\title{
Changes in Receptive Field Size in Syngap1 +/- Mouse Model of Autism Spectrum Disorder
}

\author{
Evan Anderson ${ }^{1}$, Charles A Martin ${ }^{2}$, Maria Dadarlat ${ }^{2}$ \\ ${ }^{1}$ Indiana University School of Medicine; ${ }^{2}$ Purdue University, Department of Biomedical \\ Engineering
}

\section{Background and Hypothesis:}

Autism spectrum disorder (ASD) is a form of intellectual disability with impairments in social functioning and cognition. Mutations within the SynGAP1 gene are associated with ASD due to over activation of RAS-GTP causing insertion of AMPA receptors onto the post synaptic membrane, and thus early maturation of dendrites. These mutations lead to an excitatory/inhibitory imbalance within the brain, and patients often present with intellectual disability, seizures, and issues of cognition. Research has shown that Syngap1+/- mice have decreased cortical gray matter brain volume throughout areas involved in the visual system pathway. However, hierarchal visual processing has not been well characterized in a Syngap1 $+/$ - mouse model of autism.

\section{Project Methods:}

Using 64 and 128 channel microelectrodes, we recorded the neural activity within V1 of four mice. Neural activity was recorded in response to visual stimuli - testing receptive field size between two wild-type and two Syngap1 +/- mice. Data was run through spike sorting algorithms to identify neurons. Receptive field area for each neuron was then calculated and compared between the two genotypes.

\section{Results:}

The receptive field areas of Syngap $1+/$ - mice were statistically larger $(p<0.01)$ compared to wild type mice. Syngap1 +/- mice had a mean receptive field area of 450.5 visual degrees $( \pm 443.7)$ and WT mice had a mean receptive field area of 261.1 visual degrees $( \pm 187.21)$. Most of the neurons within Syngap $+/$ - had no distinct receptive field, $67.9 \%$, while only $25.7 \%$ of wild type neurons lacked a distinct receptive field.

\section{Conclusion and Potential Impact:}

Overall, Syngap1 +/- mice had larger, and less distinct receptive fields compared to wild type mice. Deficiencies in the Syngap1 protein may impair refinement of visual stimuli. Understanding the mechanism of response of missing SynGAP1 can inform directed therapies and interventions to treat patients with the missing gene and manage their intellectual disability. 